\title{
Development of an anatomically correct mouse phantom for dosimetry measurement in small animal radiotherapy research
}

\author{
George Soultanidis $^{1,2}$, Anna Subiel ${ }^{3}$, Isaline Renard ${ }^{7}$, Anna Merle Reinhart ${ }^{4}$, \\ Victoria L Green ${ }^{1}$, Uwe Oelfke ${ }^{4}$, Stephen J. Archibald ${ }^{7}$, John Greenman ${ }^{1}$, Amanda \\ Tulk $^{5}$, Adrian Walker ${ }^{6}$, Giuseppe Schettino ${ }^{3,9}$ and Christopher J Cawthorne ${ }^{7,8^{*}}$ \\ ${ }^{1}$ Department of Biomedical Sciences, University of Hull, Hull, United Kingdom \\ ${ }^{2}$ Translational and Molecular Imaging Institute, Icahn School of Medicine at Mount Sinai, New York, \\ United States of America \\ ${ }^{3}$ Medical Radiation Science, National Physical Laboratory, Teddington, United Kingdom \\ ${ }^{4}$ Radiotherapy Physics Modelling Team, Institute of Cancer Research, Sutton, United Kingdom \\ ${ }^{5}$ Xstrahl, Camberley, United Kingdom \\ ${ }^{6}$ Leeds Test Objects, Boroughbridge, United Kingdom \\ ${ }^{7}$ PET Research Centre, University of Hull, Hull, United Kingdom \\ ${ }^{8}$ Nuclear Medicine and Molecular Imaging/Molecular Small Animal Imaging Centre, KU Leuven, \\ Leuven, Belgium \\ ${ }^{9}$ Department of Physics, University of Surrey, Guilford, United Kingdom
}

This is the accepted manuscript of an article published in Physics in Medicine and Biology at https://doi.org/10.1088/1361-6560/ab215b

*E-mail: christopher.cawthorne@kuleuven.be

Received xxxxxx

Accepted for publication $\mathrm{xxxxxx}$

Published xxxxxx

\begin{abstract}
Significant improvements in radiotherapy are likely to come from biological rather than technical optimization, for example increasing tumour radiosensitivity via combination with targeted therapies. Such paradigms must first be evaluated in preclinical models for efficacy, and recent advances in small animal radiotherapy research platforms allow advanced irradiation protocols, similar to those used clinically, to be carried out in orthotopic models. Dose assessment in such systems is complex however, and a lack of established tools and methodologies for traceable and accurate dosimetry is currently limiting the capabilities of such platforms and slowing the clinical uptake of new approaches. Here we report the creation of an anatomically correct phantom, fabricated from materials with tissue-equivalent electron density, into which dosimetry detectors can be incorporated for measurement as part of quality control (QC). The phantom also allows training in preclinical radiotherapy planning and cross-institution validation of dose delivery protocols for small animal radiotherapy platforms without the need to sacrifice animals, with high reproducibility.

Mouse CT data was acquired and segmented into soft tissue, bone and lung. The skeleton was fabricated using 3D printing, whilst lung was created using computer numerical control (CNC) milling. Skeleton and lung were then set into a surfacerendered mould and soft tissue material added to create a whole-body phantom. Materials for fabrication were characterized
\end{abstract}


for atomic composition and attenuation for X-ray energies typically found in small animal irradiators. Finally cores were CNC milled to allow intracranial incorporation of bespoke detectors (alanine pellets) for dosimetry measurement.

Keywords: phantom, radiotherapy, 3D printing

\section{Introduction}

In the past 20 years, the delivery of radiotherapy has been revolutionized by technical developments, allowing the delivery of intensity modulated and image guided radiotherapy doses with high precision (Citrin, 2017). It is likely that further improvements in radiotherapy response will be driven by the biological optimization via the inclusion of functional imaging (Nahum and Uzan, 2012) and/or the combination of radiation dose with targeted therapies, guided by biomarker evaluation (Chen and Kuo, 2017; Kirsch et al., 2018). Central to the development of such strategies is an improved understanding of radiobiology, derived from testing in preclinical models of cancer. Recently, preclinical irradiation systems such as the Small Animal Radiotherapy Research Platform (SARRP; Xstrahl, USA) have been developed that allow delivery to in vivo cancer models using clinically relevant techniques (Wong et al., 2008; Verhaegen, Granton and Tryggestad, 2011; Lindsay et al., 2014; Tillner et al., 2014); whilst the complexity and clinical relevance of preclinical tumour models has also increased (HerterSprie et al., 2014; Willey et al., 2015; Kersten et al., 2017). Although preclinical radiotherapy treatment planning systems mirror their clinical counterparts (Verhaegen et al., 2014), standardized protocols for the assessment of delivered dose are currently lacking (Desrosiers et al., 2013; Kazi et al., 2014). In clinical environments, Codes of Practice and international recommendations clearly define protocols with which the dose delivered must be assessed and traceable to national primary standards in order to achieve the $<5 \%$ recommended by the International Commission on Radiation Units and Measurement (ICRU, 1976), however this is not the case for pre-clinical radiation biology leaving the users to wide interpretation and development of non-traceable tools and protocols based on local expertise.

Estimation of dose delivered can be performed via computer simulation, or via direct measurement. Complex computational mouse phantoms have been created based on anatomical data derived from several sources (Stabin et al., 2006; Larsson et al., 2011) to predict dose absorbed in radiosensitive structures such as the lung. Such approaches require detailed and accurate modelling of both individual radiation sources and the specific radiation setup and, furthermore, cannot be used for quality control. Physical measurements of dose absorbed are therefore required, necessitating the development of accurate phantoms. Direct incorporation of dosimetry into animals has been utilized (Kuess et al., 2014) but this does not allow the measurement of dose in orthotopic environments without animal sacrifice. Moreover, realistic and reproducible phantoms are required for inter-comparison results between institutions and radiation modalities, essential for assessing the radiobiological reproducibility that underpins translation to the clinic.

Film-based verification of dose-planning on the SARRP has recently been reported (Wang et al., 2018), as has a portal imaging approach (Anvari, Poirier and Sawant, 2018), however neither of these methods are traceable to a national dosimetry standard. Incorporation of radiochromic films into a slice phantom for the evaluation of scattered photons has also been attempted (Welch et al., 2015, 2017), however this represents a sum of 2D geometries rather than a 3D geometry.

3D printing has been utilized to obtain anatomical accuracy (Kim et al., 2014), however the lack of validation of tissue equivalency for the materials used (specifically the skull for intracranial models and the lung for genetically engineered models) suggest that the results obtained have a potential bias. Although 3D printing has been widely used in the development of tissueequivalent phantoms for clinical use (Ehler et al., 2014; Kairn, Crowe and Markwell, 2015; Mitsouras et al., 2015; Russ et al., 2015), there are few reports and validation studies in the preclinical literature (Bache et al., 2015; Zhang et al., 2018).

In this study, we created an anatomically correct mouse phantom fabricated from tissue equivalent materials that was capable of incorporating calibrated dosimeters to allow traceable dose verification. An additional aim was to enable user training in dose planning on advanced radiotherapy platforms without the need for animal sacrifice.

\section{Materials and Methods}

\subsection{Selection of tissue equivalent materials}

The fabrication scheme (Figure 1) involved the segmentation of a computed tomography (CT) scan into three main compartments (skeleton, lung and all other tissues) based on density. This was followed by 3D printing of the skeleton, 
computer numerical controlled (CNC) milling of the lung insert and casting of the body by injection into a one-piece surface topology mould. Material for fabrication was selected on the basis of tissue equivalent electron density/photon attenuation characteristics and ease of fabrication (Table 1). SB5, LN10 and WT1 were selected as ICRU reference material for bone, lung and soft tissue respectively (White et al., 1989) to which the materials used for the development of the phantom (LN330 and LN450 polyurethane foam, Accura Bluestone) were compared. Accura Bluestone was obtained from 3D systems (USA). Geodeo Crystal Clear epoxy resin was obtained from Pebeo (France). All other tissue equivalent materials were produced at Leeds Test Objects (UK) using standard methods and recipes.

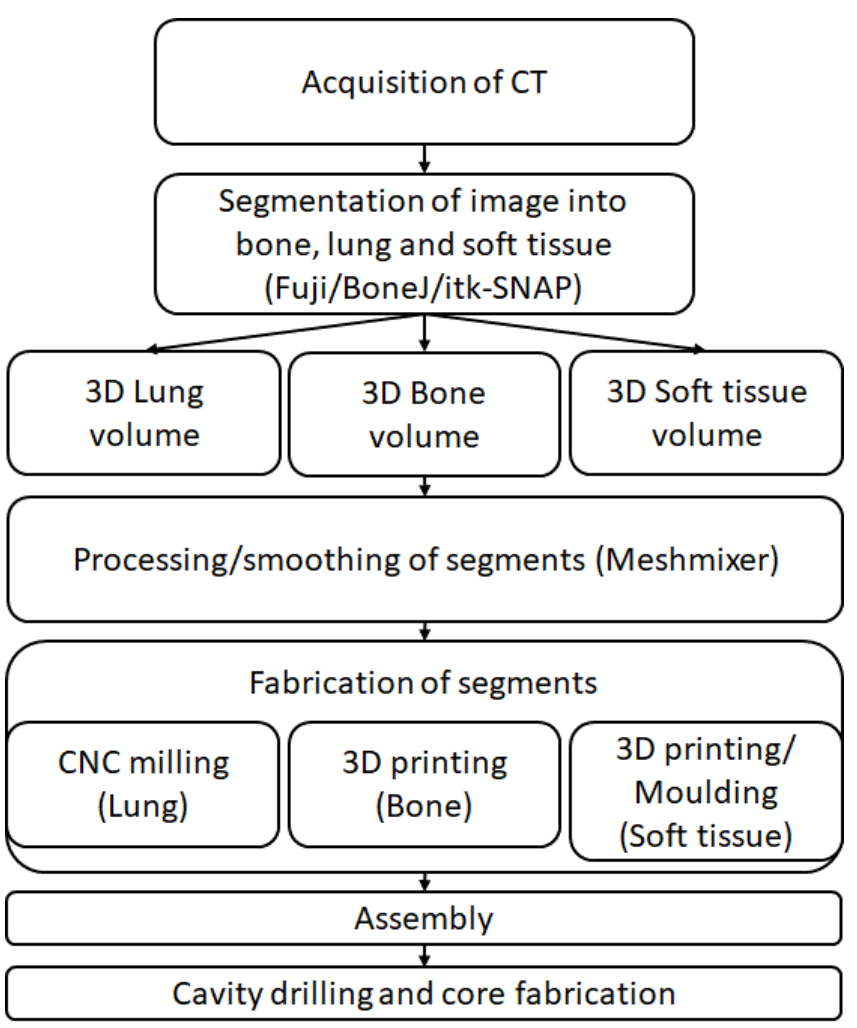

Figure 1: Fabrication scheme

Table 1: Tissue equivalent materials used in this study 


\begin{tabular}{|l|c|c|c|c|}
\hline $\begin{array}{l}\text { Commercial material } \\
\text { name }\end{array}$ & Equivalent tissue & Manufacturing method & Density & $\begin{array}{l}\text { Commercial } \\
\text { Source }\end{array}$ \\
\hline WT1* (White et al., 1989) & Soft tissue & Moulding or milling & $1.00 \mathrm{~g} / \mathrm{cm}^{3}$ & Leeds Test Objects \\
\hline $\begin{array}{l}\text { Geodeo Crystal Clear } \\
\text { epoxy Resin }\end{array}$ & Soft tissue & Moulding or milling & $1.00 \mathrm{~g} / \mathrm{cm} 3$ & Pebeo (France) \\
\hline LN10* & Lung & Moulding or milling & $0.35 \mathrm{~g} / \mathrm{cm}^{3}$ & Leeds Test Objects \\
\hline SB5* & Cortical Bone & Moulding or milling & $1.87 \mathrm{~g} / \mathrm{cm}^{3}$ & Leeds Test Objects \\
\hline LN330** & Lung (inhale) & Moulding or milling & $0.24 \mathrm{~g} / \mathrm{cm}^{3}$ & Leeds Test Objects \\
\hline LN450** & Lung (exhale) & Moulding or milling & $0.48 \mathrm{~g} / \mathrm{cm}^{3}$ & Leeds Test Objects \\
\hline Accura $^{\circledR}$ Bluestone** & Cortical Bone & 3D printing or milling & $1.78 \mathrm{~g} / \mathrm{cm}^{3}$ & 3D systems (USA) \\
\hline
\end{tabular}

*values from (White et al., 1989).

**values from manufacturer

\subsection{Characterisation of tissue equivalent materials}

\subsubsection{Elemental Analysis}

Where not reported, elemental composition was determined via Carbon, Hydrogen and Nitrogen combustion analysis (CHNN analysed), Induced Coupled Plasma Spectroscopy (ICP) and Scanning electron microscopy/Energy dispersive X-ray (SEM/EDX) analysis. Briefly, carbon, hydrogen and nitrogen percentages were determined on a minimum $1.5 \mathrm{mg}$ of sample using a Carlo Erba 1108 elemental analyser. The EA1108 is based on dynamic flash combustion and GC separation. The instrument consists of the reactor, a GC column (porapak 50-8 mesh ) and a thermal conductivity detector. The reactor is separated into 2 parts, the combustion reactor and the reduction reactor. The combustion reactor is made of translucent silica tube and is filled to a depth of $40 \mathrm{~mm}$ of quartz wool, $60 \mathrm{~mm}$ depth of universal fluorine reagent is added to the reactor tube, a further $10 \mathrm{~mm}$ of quartz wool added to the tube, on top of this $120 \mathrm{~mm}$ depth of chromium oxide is added and a further 10 $\mathrm{mm}$ of quartz wool is added. The combustion reactor sits in a furnace and when samples are to be analysed it is at $1020{ }^{0} \mathrm{C}$. The reduction tube is also a translucent silica tube, it is packed to a depth of $40 \mathrm{~mm}$ of quartz wool, $50 \mathrm{~mm}$ depth of copper oxide is added, $10 \mathrm{~mm}$ of quartz wool, followed by $280 \mathrm{~mm}$ depth of copper grains, followed by $10 \mathrm{~mm}$ quartz wool, $50 \mathrm{~mm}$ depth of copper oxide and another $10 \mathrm{~mm}$ quartz wool. The reduction reactor sits in a furnace and the temperature which is at $650{ }^{\circ} \mathrm{C}$. The GC column sits in an oven at $65{ }^{\circ} \mathrm{C}$. Results are calculated based on the known value of a standard, with all standards used traceable back to National Institute of Standards and Technology (NIST) primary standards and the analyser is checked with NIST primary standards on a regular basis to assure day-to-day accuracy of results. For ICP, a sample was weighed into a Teflon digestion vessel and $1 \mathrm{ml}$ Hydrochloric acid and $3 \mathrm{ml}$ nitric acid added. Reagents were from Romil (UK), and were SpA trace elemental grade. The vessel was sealed and probes installed to measure the temperature and pressure in the XP1500 type vessel for the microwave digestion system (CEM MARS, Milton Keynes, UK). The digestion was heated to $200^{\circ} \mathrm{C}$ in 15 minutes, retained at $200^{\circ} \mathrm{C}$ for 20 minutes then left to cool. The digest was then washed into a tared sample vial to a final weight 23.5420g with water (Elga Purelab Flex, 18Megohm.cm conductivity). Analysis was carried out on a Perkin Elmer Optima 5300DV emission ICP instrument following a standard procedure for the analysis of 69 elements against a 10ppm multi-bottle calibration set. The analysis was calibrated against an external Certified Reference digest of estuarine sediment, from Greyhound (UK). Measurements were made in duplicate and several blank 2\% nitric acid measurements allowed the estimation of real limits of detection for each element to be made. The measured ppm concentrations in the digest were calculated back to the original solid powder by multiplying the measured values by the ratio of digest weight to sample weight, then converted to \%w/w. For SEM/EDX, samples were attached to standard $25 \mathrm{~mm}$ diameter aluminium stubs. As the samples were not coated with either carbon or gold and therefore electrically nonconductive, the SEM was operated in Variable Pressure Mode. The relatively high SEM sample chamber pressure in this 
mode of operation ensures the samples do not retain an electrical charge from the electron beam. The chamber pressure was $40 \mathrm{~Pa}$ - normal high vacuum operation is performed at a pressure of better than $0.0001 \mathrm{~Pa}$. The samples were imaged using the Backscattered Electron Detector at an electron beam accelerating voltage of 20KV and probe current of 500pA. A representative area (approximately $1 \mathrm{~mm} \times 0.75 \mathrm{~mm}$ ) was selected from each sample and an elemental spectrum produced. Also, six smaller randomly selected areas on each sample were scanned and quantitative analyses produced, complete with statistics. This technique produced much more useful elemental analyses than the graphical spectra alone.

Electron density was calculated with the use of the following equation,

$$
\rho_{e}=\rho_{m} \cdot N_{A} \cdot\left(\frac{Z}{A}\right)
$$

Where,

$$
\frac{Z}{A}=\sum_{i} a_{i}\left(\frac{Z_{i}}{A_{i}}\right)
$$

$\rho_{\mathrm{m}}$ is the density of the material, $\mathrm{N}_{\mathrm{A}}$ represents the Avogadro's number, $a_{i}$ is the fraction by weight of the ith element of atomic number $Z$ and atomic weight $A$.

\subsubsection{Measurement of attenuation characteristics}

Attenuation of a $100 \times 100 \times 5 \mathrm{~mm}^{3}$ slab of each material (SB5, Accura Bluestone, LN10, PU330 \& WT1) was measured at the National Physical Laboratory (NPL) (UK) using a calibrated ionization chamber (TW30012 Farmer type, traceable to the UK primary standard $300 \mathrm{kV}$ free air chamber at the NPL). Reference X-ray beams with a Half Value Layer (HVL) between 0.5-4mm Cu were used as these characteristics were identified as common for radiobiological studies in a national capability mapping exercise carried out as part of the wider project (http://www.npl.co.uk/science-technology/radiation-dosimetry/preclinical-dosimetry-service/). The irradiation setup consisted of a $1 \mathrm{~cm}$ buildup/20 $\mathrm{cm}$ backscatter blocks of WT1 between which material for testing was inserted (Figure 2). The source to source distance (SSD) has been set to $75 \mathrm{~cm}$ which resulted in the field size at the front face of the phantom of $7 \mathrm{~cm}$. 


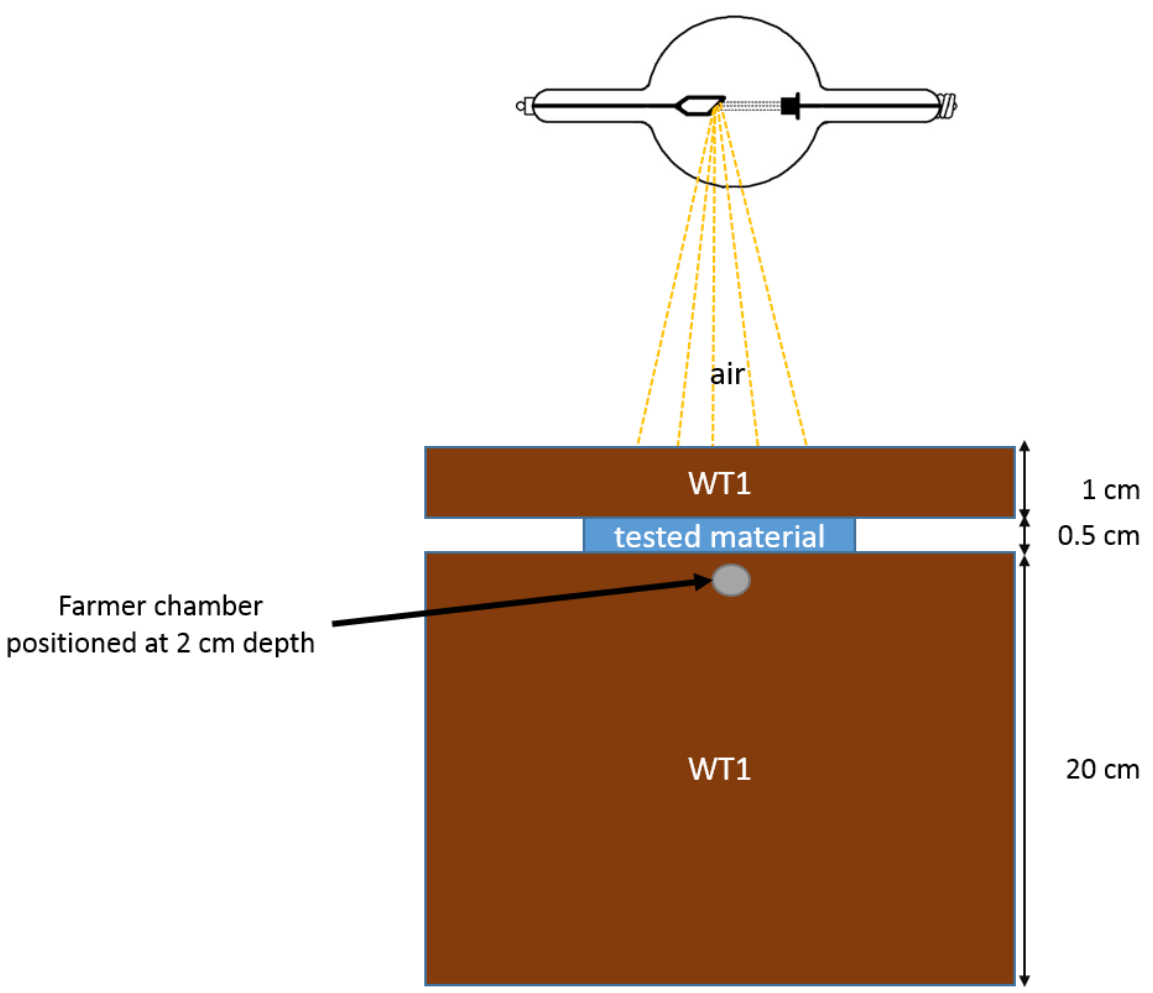

Figure 2: Experimental setup for attenuation measurements

Relative attenuation of each uncharacterised material to its ICRU-defined counterpart was calculated according to: $\%$ difference $=(1-[$ Dose $]($ tested material $) /[$ Dose $]($ ICRU reference material $) \times 100 \%$

Beam qualities are reported in Table 2.

Table 2: Medium-energy X-ray qualities used for therapy level calibrations at NPL.

\begin{tabular}{|c|c|c|c|c|}
\hline \multirow{2}{*}{ HVL $[\mathrm{mm} \mathrm{Cu}]$} & \multirow{2}{*}{$\begin{array}{c}\text { Nominal generating tube } \\
\text { potential }[\mathrm{kV}]\end{array}$} & \multicolumn{3}{|c|}{ Additional filtration } \\
\cline { 3 - 5 } & 135 & - & 0.27 & {$[\mathrm{~mm} \mathrm{Sn}]$} \\
\hline 0.5 & 180 & - & 0.54 & 1.2 \\
\hline 1 & 220 & - & 1.40 & 0.9 \\
\hline 2 & 280 & 1.5 & 0.26 & 1.0 \\
\hline 4 & & &
\end{tabular}

\subsection{Fabrication of mouse phantom}

\subsubsection{CT scanning}

Animal studies were performed in accordance with the United Kingdom's Guidance on the Operation of Animals (Scientific Procedures) Act 1986 and within guidelines set out by the United Kingdom National Cancer Research Institute Committee on


27days; weight 28g) was purchased from Charles River Laboratories (Charles River, UK) and anaesthetized using a 5\% isoflurane/oxygen mixture. It was then placed into an animal imaging cell (Minerve, France); temperature and respiratory rate were monitored throughout imaging using a dedicated monitor (SA Instruments, USA). CT scans were acquired using the Sedecal SuperArgus PET/CT scanner (40kV, $140 \mu \mathrm{A}, 360$ projections, 8 shots, $200 \mu \mathrm{M}$ pixel size). Phantom acquisitions were 
made using the same settings. Animal and phantom images were also obtained on the small animal radiotherapy research platform (SARRP) (Xstrahl, UK) using $60 \mathrm{kVp}, 0.8 \mathrm{~mA}, 1 \mathrm{~mm} \mathrm{Al} \mathrm{filter.}$

\subsubsection{Image segmentation and display}

Images were segmented using Fiji (Schindelin et al., 2012) and the segmented data used as an input for the BoneJ plugin. Thresholds were set to delineate the entire skeleton and whole body whilst lungs were segmented semi-automatically using itkSNAP (Yushkevich and Gerig, 2017) by placing seeds within the lung region and executing the evolution algorithm (competition force set at 1, smoothing set to 0.2). Stereolithography (STL) files were then exported using a greyscale window. Meshmixer (Autodesk®Inc., USA) was used to produce the final skeleton volume, after further smoothing and thresholding to create a continuous form. In figure 3A, we rendered the different components, using Blender, an open source 3D modeling software.

\subsubsection{Density analysis}

A reference CT phantom containing reference material representing Brain, Adipose Tissue, Breast, Solid Water, Liver, Inner Bone, Bone and Cortical Bone ( $\mu$ CT calibration phantom, SmART Scientific Solutions B.V., Netherlands) was scanned using the same settings as for the mouse (described above). Regions of interest (ROIs) were drawn around the centre of each insert and a calibration curve was generated. Scan units were converted to density using the calibration curve (Saw et al., 2005).

\subsubsection{Phantom fabrication methodology}

3D printing of mouse skeleton using Accura Bluestone was carried out with a stereolithography (SLA) ProX 800 Printer (3D systems Inc., USA). The maximum print resolution was $4000 \mathrm{DPI}^{2}$ with a minimum voxel size of $0.4 \mathrm{~mm}^{3}$, these parameters were applied when processing STL files. Lung insert tool paths were generated using Autodesk ${ }^{\circledR} F u s i o n ~ 360$ (Autodesk ${ }^{\circledR}$, USA) and exported to a Datron M7 high-speed CNC machine (Datron Dynamics Inc., USA) for processing of LN330 or LN10 blocks (without coolant).

A solid phantom was created using the surface rendering of the CT scan and from this a silicone mould was created. Skeleton and lung volumes were assembled at the correct anatomical locations and water-equivalent material (transparent Epoxy resin or WT1) was introduced. Illustrative skeleton, lung inserts and final phantoms are shown in figure 3. To facilitate insertion and removal of alanine pellets, a $5.2 \mathrm{~mm}$ cylindrical void was milled into the skull after fabrication using a custom jig; a removable skull ‘cap' for insertion over the pellet was fabricated using a combination of milling and 3D printing (Figure 4).

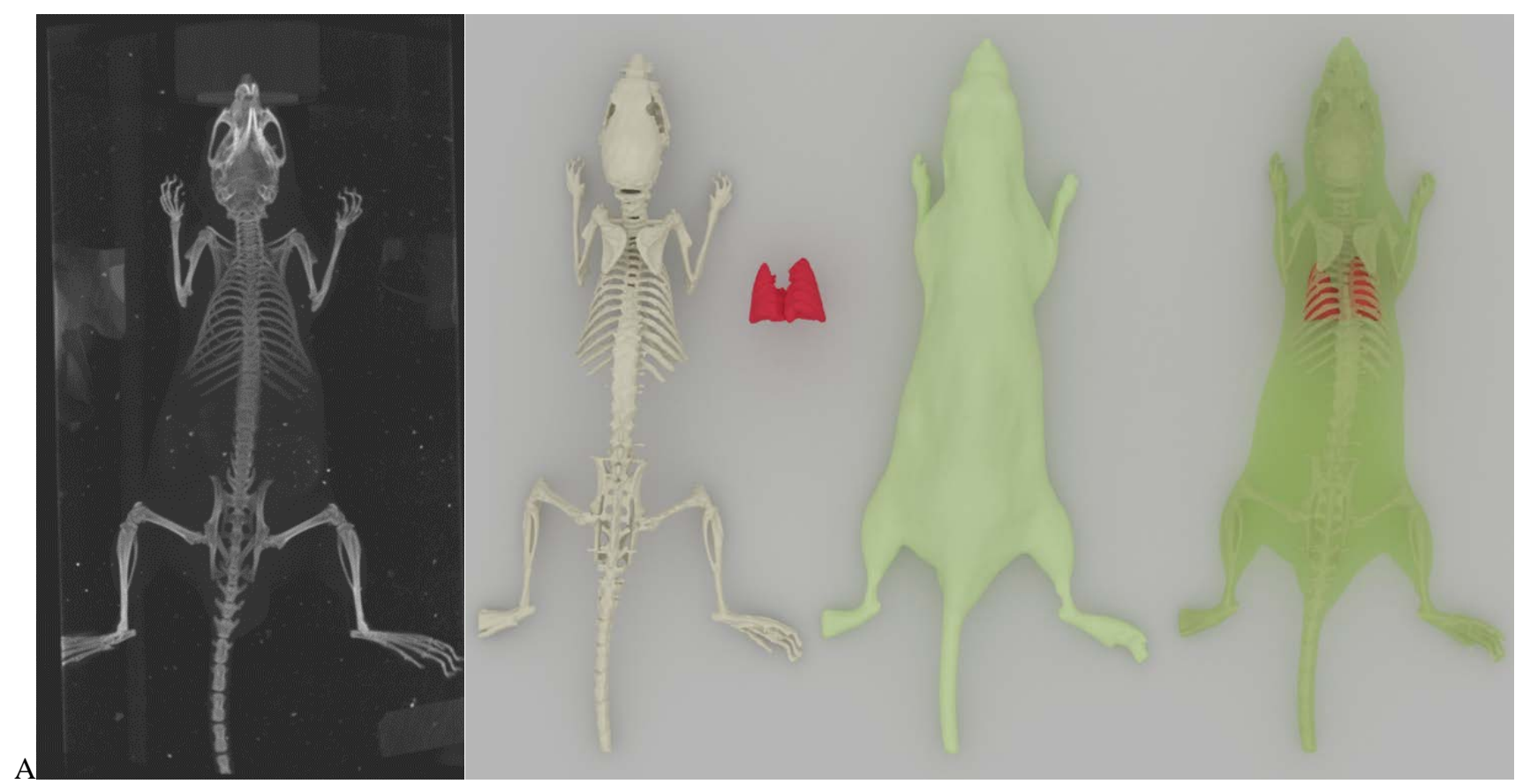




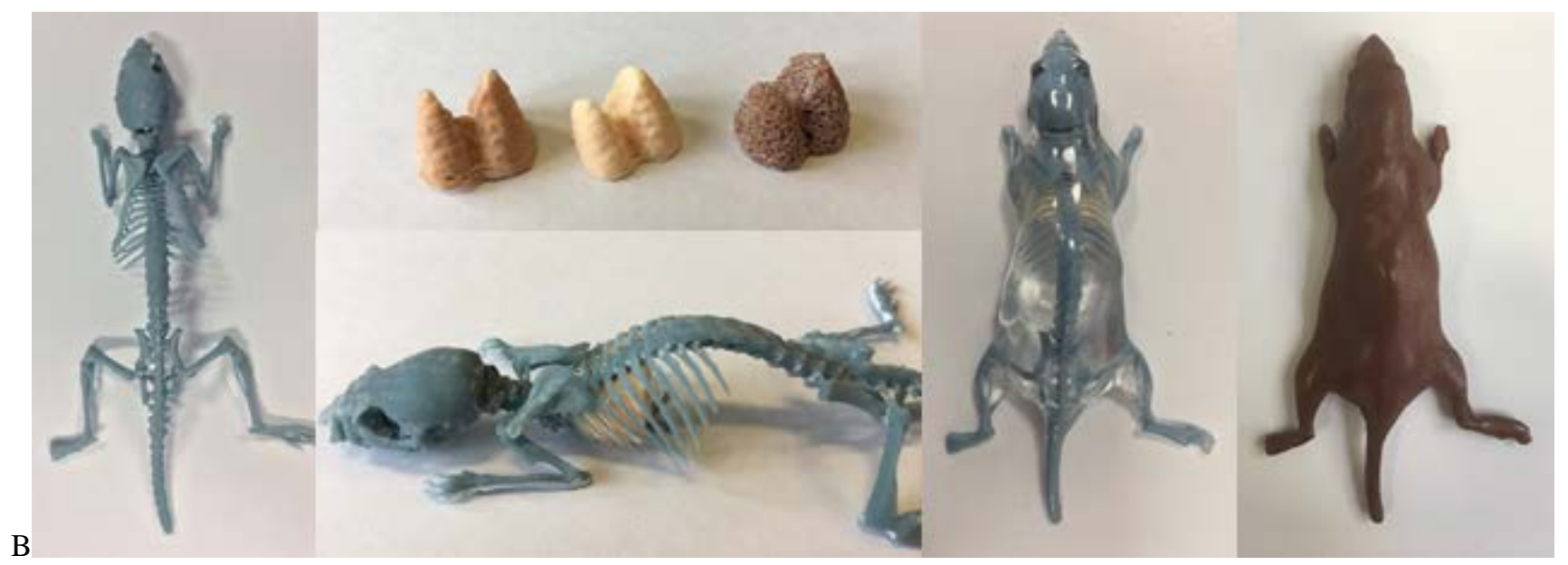

Figure 3: Maximum intensity projections from CT scan of original animal and resulting segmentation illustrating bone, lung and soft tissue components separately and fused (Left to right respectively; A). Printed skeleton, milled lung inserts (LN330, LN450 and LN10), assembled skeleton/lung construct before whole body casting to create final phantom (B). Transparent Epoxy resin phantom allows internal structure to be seen for demonstration.
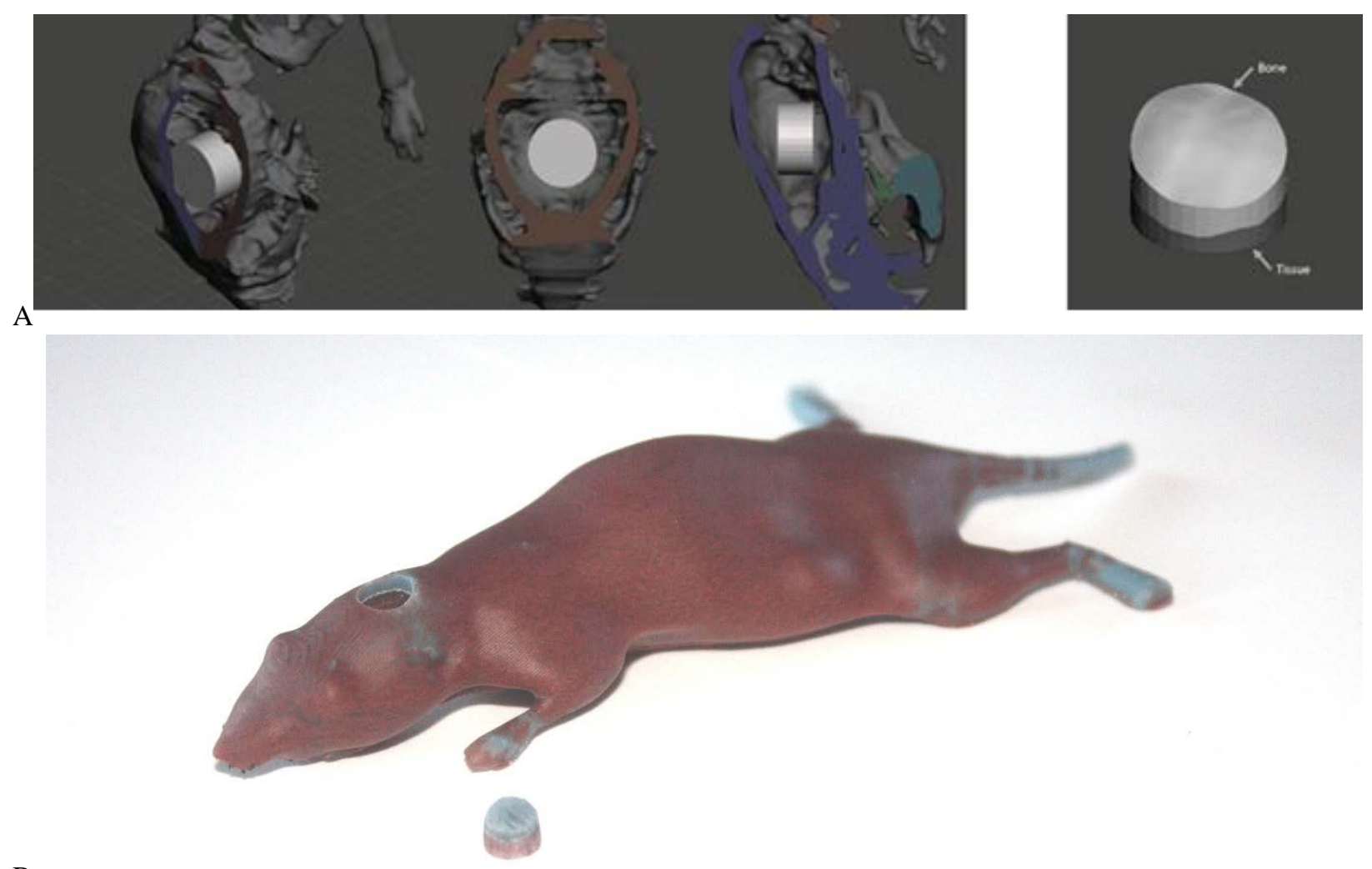

B

Figure 4: Design of cavity and core insert for intracranial incorporation of alanine pellet into skull with skull 'cap', visualised in Meshmixer (A) and cored phantom with insert (B).

\section{Results}

\subsection{Elemental composition analysis}

Table 3: Elemental analysis

\begin{tabular}{|c|c|c|c|c|c|c|}
\hline \multirow{2}{*}{$\begin{array}{c}\text { Tissue } \\
\text { substitute }\end{array}$} & \multicolumn{5}{|c|}{ Elemental composition (percentage by mass) } & Electron density \\
\hline & $\mathrm{H}$ & $\mathrm{C}$ & $\mathrm{N}$ & $\mathrm{O}$ & Others & (electrons/g) $\times 10^{23}$ \\
\hline
\end{tabular}




\begin{tabular}{|c|c|c|c|c|c|c|}
\hline $\begin{array}{c}\text { Accura } \\
\text { Bluestone }\end{array}$ & 3.1 & 22.8 & $<0.1$ & 45.2 & $25.3 \mathrm{Si}, 0.4 \mathrm{Cl}, 0.49 \mathrm{Al}, 2.16 \mathrm{~F}, 0.23 \mathrm{Sb}$ & 7.07 \\
\hline SB5 & 2.6 & 30.6 & 1.0 & 38.9 & $0.1 \mathrm{Cl}, 26.8 \mathrm{Ca}$ & 5.68 \\
\hline LN330 & 6.21 & 59.0 & 4.65 & 28.1 & $0.3 \mathrm{Ca}, 0.5 \mathrm{Mg}, 0.3 \mathrm{Si}, 0.6 \mathrm{Cl}$ & 0.73 \\
\hline LN10/75 & 8.4 & 60.4 & 1.7 & 17.3 & $11.4 \mathrm{Mg}, 0.7 \mathrm{Si}, 0.1 \mathrm{Cl}$ & 0.81 \\
\hline WT1 & 8.1 & 67.2 & 2.4 & 19.9 & $0.1 \mathrm{Cl}, 2.3 \mathrm{Ca}$ & 3.25 \\
\hline
\end{tabular}

Elemental analysis of Accura Bluestone and LN330 indicated similar composition to the ICRU reference materials SB5 and LN10/75 respectively (obtained from (White et al., 1989)).

\subsection{Attenuation characteristics}

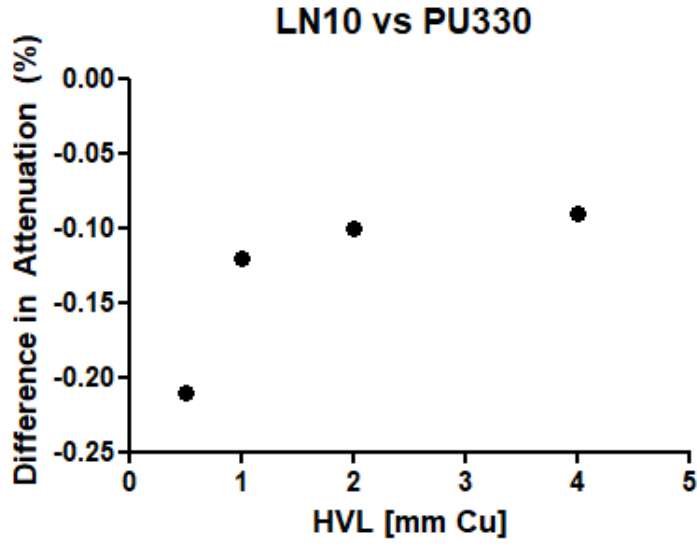

A

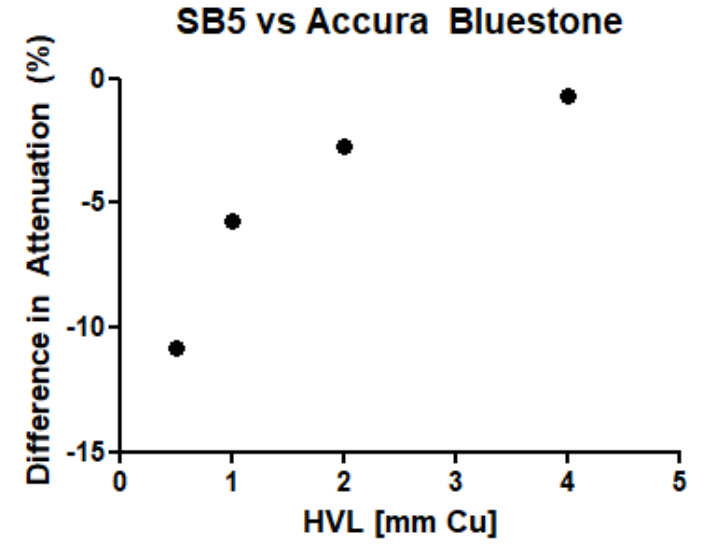

B

Figure 5: Difference in attenuation between (A) Accura Bluestone and SB5 and (B) LN330 and LN10 as a function of beam quality (mm Cu HVL).

Attenuation properties of Accura Bluestone and LN330 were broadly comparable to SB5 and LN10 (Figure 5). For Accura Bluestone/SB5, data indicate a $10 \%$ difference in attenuation for very soft beams ( $0.5 \mathrm{~mm} \mathrm{Cu} \mathrm{HVL)}$; this difference decreases rapidly to $5 \%$ for $1 \mathrm{~mm} \mathrm{Cu} \mathrm{HVL}$ and to less than 1\% for $4 \mathrm{~mm} \mathrm{Cu} \mathrm{HVL}$. For PU330/LN10, the difference in attenuation was less than $1 \%$ at all HVL thicknesses (Figure 5).

\subsection{Assessment of phantom radiotherapy dose planning}

The ability to support treatment planning was initially assessed on the small animal radiotherapy research platform using phantoms incorporating lung material fabricated from LN10 and LN330. Although treatment planning could be carried out to the phantom brain for both phantoms, the heterogeneous structure and inclusion of air voids in LN10 precluded dose planning to the lung where this material had been used. Dose planning to the LN330 lung was successful (Figure 6). 


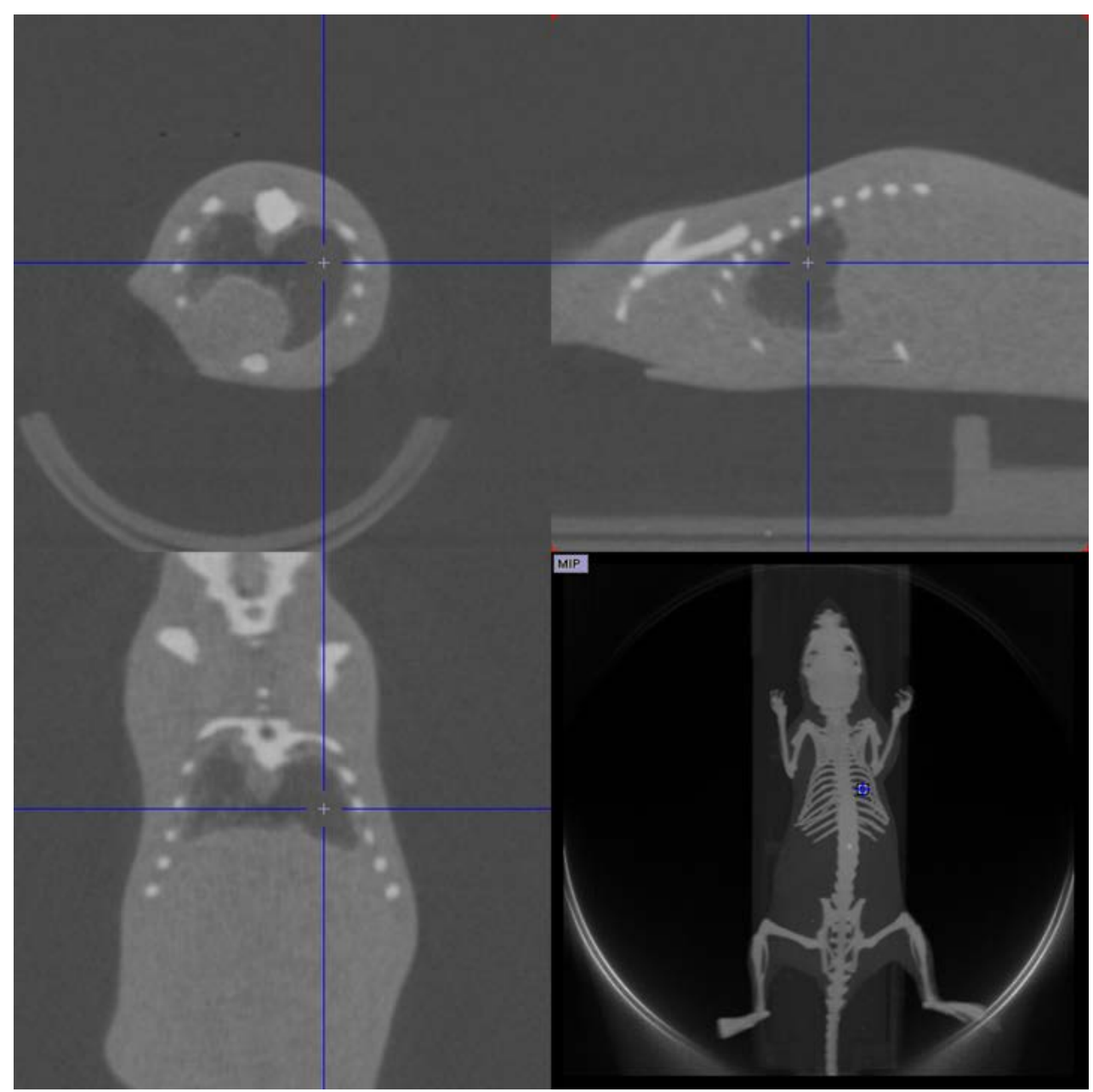

Figure 6: Transaxial, sagittal and coronal single slices through phantom lung and associated maximum intensity projection from SARRP CT scan.

\subsection{Evaluation of phantom anatomical accuracy and attenuation on CT}

Segmentation volumes were compared on CT scans of the original mouse and the mouse phantom (Table 3); CT derived density was also compared (Table 4). The fabricated phantom was similar with respect to segmented tissue volumes and density to the original segmented files. 


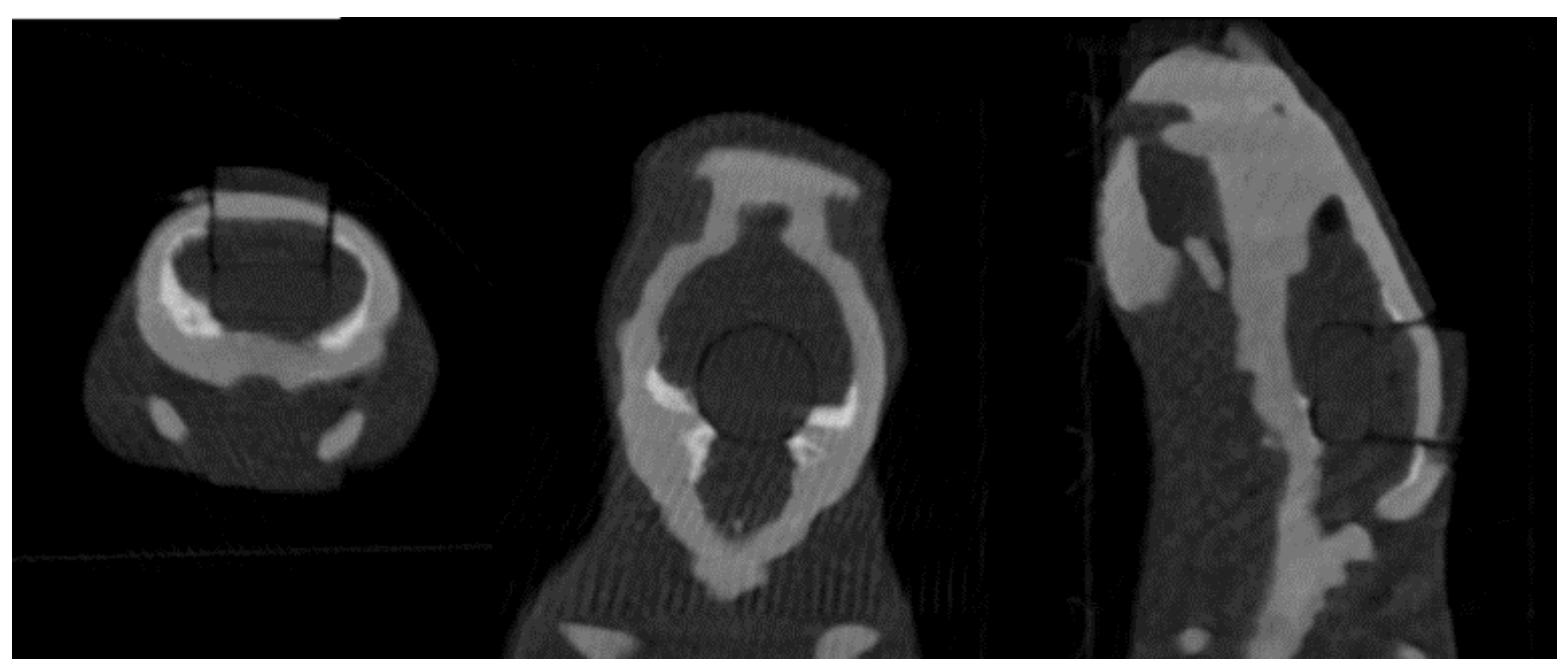

Figure 7: Transaxial, Coronal and Sagittal sections from CT of final cored phantom, centred on inserted alanine pellet.

Table 4: Segmented volumes derived from original mouse and derived phantoms

\begin{tabular}{|c|c|c|c|}
\hline & \multicolumn{3}{|c|}{ Volume $\left(\mathrm{mm}^{3}\right)$} \\
\hline & Bone & Tissue & Lung \\
\hline Mouse & 2107.1 & 21389.6 & 457.0 \\
\hline Phantom 3D model (in silico, via MeshMixer) & 2597.2 & 17943.0 & 454.9 \\
\hline Phantom (WT1) & 3203.0 & 18132.2 & 409.8 \\
\hline
\end{tabular}

Table 5: Comparison of average mouse and phantom segment densities as measured by CT (relative to water electron density, $\left.\rho^{\mathrm{w}} \mathrm{e}\right)$.

\begin{tabular}{|c|c|c|}
\hline & Mouse & Phantom (WT1, PU330) \\
\hline Bone & 1.39 & 1.42 \\
\hline Tissue & 1.00 & 1.01 \\
\hline Lung & 0.68 & 0.28 \\
\hline
\end{tabular}

\subsection{Incorporation}

\section{of dosimetry}

An alanine pellet (as used by the NPL dosimetry service: http://www.npl.co.uk/science-technology/radiationdosimetry/products-and-services/alanine-reference-dosimetry-service-for-radiotherapy) of $5 \mathrm{~mm}$ diameter and $2.5 \mathrm{~mm}$ thickness was incorporated into the skull cavity as described above (Figures 4 and 7). Optimum cavity size for ease of insertion and removal using a vacuum suction tool was established as $5.2 \mathrm{~mm}$. Reproducibility of dose measurements (including alanine insertion/removal and phantom positioning in the radiation field) was assessed by irradiating alanine pellets in the phantom under identical conditions $(\mathrm{n}=3$ ) using a $0.5 \mathrm{~mm}$ Cu HVL, $7 \mathrm{~cm}$ diameter X-ray beam (described in section 2.2.2) with nominal doses ranging from $10-50 \mathrm{~Gy}$. The average standard deviation of the measured dose was $0.58 \%$ with a maximum spread of $1.8 \%$. These results are well within the uncertainty in absolute dose measurement for alanine of $2.2 \%$ at 2 sigma.

\section{Discussion}

Currently, standardized protocols and tools for dose assessment (routine in clinical practice) are lacking in preclinical radiotherapy. Here we report a tissue-equivalent, anatomically correct phantom that can incorporate established traceable dosimeters for quality control, and can be used as a training tool for dose planning on advanced small animal irradiators. The phantom is currently designed to host standard alanine pellets used by NPL for postal dosimetry service (i.e. $5 \mathrm{~mm}$ diameter, 
$2.5 \mathrm{~mm}$ thick) as the majority of pre-clinical investigations use $>5 \mathrm{~mm}$ radiation beams. Small alanine pellets can be used and the phantom adapted if necessary. The traceability of the dosimetry methodology will be addressed in a follow up study.

The phantom is robust and commercially obtainable from CT datasets by Leeds Test Objects (UK). The method can thus be readily adapted and optimised with computer aided design software to produce phantom 'avatars' for dose planning in either advanced genetically engineered mouse models of cancer or those based on slow-growing patient-derived xenografts; the STL files generated as part of the manufacturing process are also suitable for the creation of computational phantoms using GATE. GATE can also be used to simulate not only CT acquisitions but also radiotherapy treatment planning to calculate dose distributions, a necessary tool for further evaluation once the radiation source details are known. Although the phantom does not incorporate respiratory motion which introduces considerable variability in thoracic irradiation protocol, current approaches seek to correct for this in live animals via gating, effectively irradiating when movement is minimised (Hill and Vojnovic, 2017). The current version of the phantom uses CNC manufacturing for the lungs as this is the best manufacturing option for the identified lung-equivalent materials. Current activities at NPL and Christie hospital are ongoing to identify 3D printable tissue equivalent materials (including lung) which could then be also employed to improve the mouse phantom reported in this study by 3D printing the lung volumes.

As can be seen from the CT projections, the phantom anatomy closely resembles that of the source animal. Although the skeleton was segmented to support fabrication, and it is thus thicker than that found in vivo, it still achieves the aim of allowing planned doses to be compared with actual ones in a realistic setting, as well as enabling training in radiotherapy delivery to be carried out without animal sacrifice; it will also be possible to compare GATE simulations of original vs. phantom CT scans to assess the impact of these differences in future work.

CT scans indicate a $<2 \%$ difference in the electron density for the skeleton which compares well with the $<10 \%$ in dose attenuation seen at the softer X-ray beam used $(0.5 \mathrm{~mm} \mathrm{Cu} \mathrm{HVL})$. Reproducibility of dose delivered to the alanine pellet is well within the accuracy required for radiobiological investigations whilst the feasibility of using alanine for absolute dose measurements is well established (Burns et al., 2011). Importantly the X-ray beam used for attenuation measurements in this study is similar to those commonly delivered by pre-clinical radiotherapy units.

\section{Conclusion}

In this study we report the fabrication of an anatomically correct, tissue-equivalent mouse phantom that can incorporate traceable dosimetry to support dose delivery assessment in preclinical irradiation systems and support radiotherapy planning training on these platforms. Future work will validate dose measurement protocols and incorporate biological dosimetry into the device.

\section{Acknowledgements}

This work was supported by grants from the Engineering and Physical Sciences Research Council (EP/N510117/1) and Innovate UK (102524). We acknowledge Tony Sinclair, Bob Knight and Carol Kennedy from the Department of Chemistry at the University of Hull for photoelectron spectroscopy, inductively coupled plasma and combustion analysis respectively.

\section{References}

Anvari, A., Poirier, Y. and Sawant, A. (2018) 'Kilovoltage transit and exit dosimetry for a small animal image-guided radiotherapy system using built-in EPID’, Medical Physics. John Wiley \& Sons, Ltd, 45(10), pp. 4642-4651. doi: 10.1002/mp.13134.

Bache, S. T. et al. (2015) 'Investigating the accuracy of microstereotactic-body-radiotherapy utilizing anatomically accurate 3D printed rodent-morphic dosimeters', Medical Physics, 42(2), pp. 846-855. doi: 10.1118/1.4905489.

Burns, D. T. et al. (2011) 'Supplementary comparison CCRI(I)-S2 of standards for absorbed dose to water in ${ }^{60}$ Co gamma radiation at radiation processing dose levels', Metrologia. IOP Publishing, 48(1A), pp. 06009-06009. doi: 10.1088/00261394/48/1A/06009.

Chen, H. H. W. and Kuo, M. T. (2017) 'Improving radiotherapy in cancer treatment: Promises and challenges', Oncotarget, 8(37), pp. 62742-62758. doi: 10.18632/oncotarget.18409.

Citrin, D. E. (2017) 'Recent Developments in Radiotherapy', New England Journal of Medicine. Edited by D. L. Longo. Massachusetts Medical Society, 377(11), pp. 1065-1075. doi: 10.1056/NEJMra1608986. 
Desrosiers, M. et al. (2013) 'The Importance of Dosimetry Standardization in Radiobiology.', Journal of research of the National Institute of Standards and Technology. National Institute of Standards and Technology, 118, pp. 403-18. doi: 10.6028/jres.118.021.

Ehler, E. D. et al. (2014) 'Patient specific 3D printed phantom for IMRT quality assurance', Physics in Medicine and Biology, 59(19), pp. 5763-5773. doi: 10.1088/0031-9155/59/19/5763.

Herter-Sprie, G. S. et al. (2014) 'Image-guided radiotherapy platform using single nodule conditional lung cancer mouse models’, Nature Communications, 5(1), p. 5870. doi: 10.1038/ncomms6870.

Hill, M. A. and Vojnovic, B. (2017) 'Implications of respiratory motion for small animal image-guided radiotherapy', The British Journal of Radiology, 90(1069), p. 20160482. doi: 10.1259/bjr.20160482.

International Commission on Radiation Units and Measurements (1976) 'Determination of Absorbed Dose in a Patient Irradiated by Beams of X- or Gamma-rays in Radiotherapy Procedures', ICRU Rep. 24, Bethesda, MD. Available at: https://academic.oup.com/jicru/article-abstract/os13/1/NP/2923522 (Accessed: 3 December 2018).

Kairn, T., Crowe, S. B. and Markwell, T. (2015) 'Use of 3D Printed Materials as Tissue-Equivalent Phantoms’, in. Springer, Cham, pp. 728-731. doi: 10.1007/978-3-319-19387-8_179.

Kazi, A. M. et al. (2014) 'The MCART Radiation Physics Core’, Health Physics, 106(1), pp. 97-105. doi: 10.1097/HP.0b013e3182a2a987.

Kersten, K. et al. (2017) 'Genetically engineered mouse models in oncology research and cancer medicine.', EMBO molecular medicine. Wiley-Blackwell, 9(2), pp. 137-153. doi: 10.15252/emmm.201606857.

Khan, Faiz M. et al. (2014) 'Khan's The Physics of Radiation Therapy.’, ISBN/ISSN: 9781451182453, wolters kluwer publishing

Kim, H. et al. (2014) 'Establishing a process of irradiating small animal brain using a CyberKnife and a microCT scanner', Medical Physics, 41(2), p. 021715. doi: 10.1118/1.4861713.

Kirsch, D. G. et al. (2018) 'The Future of Radiobiology.', Journal of the National Cancer Institute. Oxford University Press, 110(4), pp. 329-340. doi: 10.1093/jnci/djx231.

Kuess, P. et al. (2014) 'Dosimetric challenges of small animal irradiation with a commercial X-ray unit', Zeitschrift für Medizinische Physik, 24(4), pp. 363-372. doi: 10.1016/j.zemedi.2014.08.005.

Larsson, E. et al. (2011) 'Monte Carlo calculations of absorbed doses in tumours using a modified MOBY mouse phantom for pre-clinical dosimetry studies’, Acta Oncologica, 50(6), pp. 973-980. doi: 10.3109/0284186X.2011.582517.

Lindsay, P. E. et al. (2014) 'Multi-institutional dosimetric and geometric commissioning of image-guided small animal irradiators’, Medical Physics, 41(3), p. 031714. doi: 10.1118/1.4866215.

Mitsouras, D. et al. (2015) 'Medical 3D Printing for the Radiologist', RadioGraphics. Radiological Society of North America , 35(7), pp. 1965-1988. doi: 10.1148/rg.2015140320.

Nahum, A. E. and Uzan, J. (2012) ‘(Radio)Biological Optimization of External-Beam Radiotherapy’, Computational and Mathematical Methods in Medicine. Hindawi, 2012, pp. 1-13. doi: 10.1155/2012/329214.

Russ, M. et al. (2015) 'Treatment Planning for Image-Guided Neuro-Vascular Interventions Using Patient-Specific 3D Printed Phantoms.', Proceedings of SPIE--the International Society for Optical Engineering. NIH Public Access, 9417. doi: 10.1117/12.2081997.

Saw, C. B. et al. (2005) 'Determination of CT-to-density conversion relationship for image-based treatment planning systems’, Medical Dosimetry, 30(3), pp. 145-148. doi: 10.1016/j.meddos.2005.05.001.

Schindelin, J. et al. (2012) 'Fiji: an open-source platform for biological-image analysis', Nature Methods, 9(7), pp. 676-682. doi: 10.1038/nmeth.2019.

Stabin, M. G. et al. (2006) 'Voxel-based mouse and rat models for internal dose calculations.', Journal of nuclear medicine : official publication, Society of Nuclear Medicine, 47(4), pp. 655-9. Available at:

http://www.ncbi.nlm.nih.gov/pubmed/16595500 (Accessed: 3 December 2018).

The Royal College of Radiologists (2015) How the next Government can improve services for cancer patients. Available at: www.rcr.ac.uk/CO_shape_(Accessed: 3 December 2018).

Tillner, F. et al. (2014) 'Pre-clinical research in small animals using radiotherapy technology - a bidirectional translational approach’, Zeitschrift für Medizinische Physik, 24(4), pp. 335-351. doi: 10.1016/j.zemedi.2014.07.004.

Verhaegen, F. et al. (2014) 'A review of treatment planning for precision image-guided photon beam pre-clinical animal radiation studies’, Zeitschrift für Medizinische Physik, 24(4), pp. 323-334. doi: 10.1016/j.zemedi.2014.02.004.

Verhaegen, F., Granton, P. and Tryggestad, E. (2011) 'Small animal radiotherapy research platforms', Physics in Medicine and Biology, 56(12), pp. R55-R83. doi: 10.1088/0031-9155/56/12/R01. 
Wang, Y.-F. et al. (2018) 'Dosimetric verification and commissioning for a small animal image-guided irradiator', Physics in Medicine \& Biology, 63(14), p. 145001. doi: 10.1088/1361-6560/aacdcd.

Welch, D. et al. (2015) 'Construction of mouse phantoms from segmented CT scan data for radiation dosimetry studies', Physics in Medicine and Biology, 60(9), pp. 3589-3598. doi: 10.1088/0031-9155/60/9/3589.

Welch, D. et al. (2017) 'Scattered Dose Calculations and Measurements in a Life-Like Mouse Phantom', Radiation Research, 187(4), pp. 433-442. doi: 10.1667/RR004CC.1.

White, D. R. et al. (1989) 'Report 44', Journal of the International Commission on Radiation Units and Measurements. Oxford University Press, os23(1), p. NP-NP. doi: 10.1093/jicru/os23.1.Report44.

Willey, C. D. et al. (2015) 'Patient-Derived Xenografts as a Model System for Radiation Research', Seminars in Radiation Oncology. W.B. Saunders, 25(4), pp. 273-280. doi: 10.1016/J.SEMRADONC.2015.05.008.

Wong, J. et al. (2008) 'High-Resolution, Small Animal Radiation Research Platform With X-Ray Tomographic Guidance Capabilities', International Journal of Radiation Oncology*Biology*Physics, 71(5), pp. 1591-1599. doi: 10.1016/j.jijrobp.2008.04.025.

Workman, P. et al. (2010) 'Guidelines for the welfare and use of animals in cancer research', British Journal of Cancer, 102(11), pp. 1555-1577. doi: 10.1038/sj.bjc.6605642.

Yushkevich, P. A. and Gerig, G. (2017) 'ITK-SNAP: An Intractive Medical Image Segmentation Tool to Meet the Need for Expert-Guided Segmentation of Complex Medical Images’, IEEE Pulse, 8(4), pp. 54-57. doi: 10.1109/MPUL.2017.2701493. Zhang, H. et al. (2018) 'Fabrication of an anthropomorphic heterogeneous mouse phantom for multimodality medical imaging’, Physics in Medicine \& Biology. IOP Publishing, 63(19), p. 195011. doi: 10.1088/1361-6560/aadf2b. 